\title{
Introduction to the Special Issue on Crowd in Intelligent Systems
}

KUAN-TA CHEN, Academia Sinica, Taiwan

OMAR ALONSO, Microsoft, USA

MARTHA LARSON, Delft University of Technology, Netherlands

IRWIN KING, The Chinese University of Hong Kong, Hong Kong

Crowdsourcing involves outsourcing tasks to a large number of people with unspecified skills and abilities. Because crowdsourcing leverages human intelligence, it is a wellsuited strategy to address problems that are difficult for computers. In recent years, there have been compelling demonstrations that information or judgments collected from the crowd can, when appropriately aggregated, be productively applied in a range of areas from knowledge management to scientific inquiry. Effectively, crowdsourcing extends the reach of intelligent systems by filling the gap between artificial intelligence and human intelligence with the wisdom of a crowd.

This special issue contributes to addressing key research questions centered on the role of the crowd in intelligent systems, with the aim of promoting balanced and effective combinations of artificial and human intelligence. The articles in this special issue are positioned in the intersection of crowdsourcing, intelligent systems, and realworld applications. Topically, the articles interlock, but for the purposes of providing a structured overview, we divide them into three clusters.

The first cluster addresses theoretical aspects of crowdsourcing in intelligent systems, focusing on incentives, as well as on methodologies and tools. "Incentives for Effort in Crowdsourcing Using the Peer Truth Serum," by G. Radanovic, B. Faltings, and R. Jurca, proposes a game mechanism that creates incentives for crowdworkers to engage seriously with the tasks that they carry out. "A Game-Theory Approach for Effective Crowdsource-Based Relevance Assessment," by Y. Moshfeghi, A. F. Huertas Rosero, and J. M. Jose, studies gamification in crowdsourcing in the context of relevance assessments. The article describes a game framework where competition among workers is used as incentive. "PPLib: Towards the Automated Generation of Crowd Computing Programs Using Process Recombination and Auto-Experimentation," by P. de Boer and A. Bernstein, presents a methodology and program library that allows for the automated recombination of known processes stored in a repository. "Crowdsourcing Human Annotation on Web Page Structure: Infrastructure Design and Behavior-Based Quality Control,” by S. Han, P. Dai, P. Paritosh, and D. Huynh, describes the design and implementation of Wernicke, a crowdsourcing tool that collects behavioral features and use these features to predict annotation quality. The focus is then on workers' behavioral signals instead of just past performance.

The second cluster is dedicated to approaches that use crowdsourcing to improve information systems or create resources. "Using the Crowd to Improve Search Result Ranking and the Search Experience," by Y. Kim, K. Collins-Thompson, and J. Teevan, explores crowdsourcing approaches aimed at improving the effectiveness of search systems. The article considers the tradeoff between the extra time required if the crowd is in the loop and provides suggestions on how to most appropriately incorporate the crowd. "Crowdsourcing Empathetic Intelligence: The Case of the Annotation of EMMA Database for Emotion and Mood Recognition," by C. Katsimerou, J. Albeda, A. Huldtgren, I. Heynderickx, and J. A. Redi, discusses how crowdsourcing can be used

2016 Copyright is held by the owner/author(s).

2157-6904/2016/07-ART44

DOI: http://dx.doi.org/10.1145/2920522

ACM Transactions on Intelligent Systems and Technology, Vol. 7, No. 4, Article 44, Publication date: July 2016. 
as a reliable tool for annotating an affective database. The new database called EMotion and Mood Annotations (EMMA) is suited for the study of automatic emotion and mood recognition on the basis of videos of the upper/entire body and Kinect data. The authors describe control mechanisms for identifying good judgments and the final reliability of the data set. "Leveraging Human Computations to Improve Schematization of Spatial Relations from Imagery," by H. Rao, S.-W. Huang, and W.-T. Fu, proposes mobilizing the crowd to generate two-dimensional schematic maps from a set of pictures of an indoor environment, which has been a challenging and active research topic in the field of computer vision and geo-sensing. Two innovations in task design are proposed to improve the quality of the generated schematic map and are shown to be effective in sensitizing crowdworkers to relative object locations and emphasized dimensions to improve the accuracy of location and distance perception.

The third cluster is devoted to applications by which crowdsourcing serves the benefit of industry or society. "Using Crowdsourcing for Scientific Analysis of Industrial Tomographic Images,” by C. Chen, P. Woźniak, A. Romanowski, M. Obaid, T. Jaworski, J. Kucharski, K. Grundzień, S. Zhao, and M. Fjeld, presents a crowdsourcing system designed to detect tracer particles in industrial tomographic images and applies it to the problem of bulk solid flow in silos, as images from silo sensing systems cannot be adequately analyzed using the current computational methods. Crowdsoucing makes the system scalable and economical and, at the same time, supports the analysis and understanding of flow phenomena and related problems. "A Crowd-Powered System for Fashion Similarity Search," by T. Semertzidis, J. Novak, M. Lazaridis, M. Melenhorst, I. Micheel, D. Michalopoulos, M. Böckle, M. G. Strintzis, and P. Daras, describes a crowdsourcing system for fashion similarity search for use by the fashion industry. A core component of the proposed system is a machine-crowd workflow that supports tasks requiring subjective judgements. "Rapid Low-Cost Virtual Human Bootstrapping via the Crowd," by M. Borish and B. Lok, discusses the contribution that crowdsourcing can make to the rapid design of virtual human systems that can engage in question-answer chat. Such systems are important training tools and need to be developed rapidly in cases of emergy. The article focuses on systems for training medical personnel. "CITY FEED: A Pilot System of Citizen-Sourcing for City Issue Management," by L. You, G. Motta, K. Liu, and T. Ma, is devoted to cases in which the crowd is a group of citizens actively interacting and collaborating with city authorities. The article introduces a framework that describes citizen-sourcing applications in terms of their maturity and an example system.

In sum, this special issue covers a wide range of computing-related topics bringing together multiple disciplines and cross-cutting research and industry. However, crowdsourcing in intelligent systems is a relatively young field of research, and we are still far from achieving ideal human-machine integration. It is our hope that the articles brought together here will stimulate and support further work on crowdsourcing and on the many opportunities that combine human and artificial intelligence.

This special issue benefited from the contributions of many authors as well as from the effort and expertise of a large number of reviewers, with both groups drawn from many diverse disciplinary backgrounds and countries all over the world. We thank them for their time and dedication and appreciatively acknowledge the support of the ACM TIST editorial board. 